\title{
Equilibrium analysis in the parking search game with heuristic strategies
}

\author{
Evangelia Kokolaki and Ioannis Stavrakakis \\ Department of Informatics and Telecommunications, National \& Kapodistrian University of Athens, Greece \\ Email: \{evako, ioannis\}@di.uoa.gr
}

\begin{abstract}
The tremendous growth of urbanization calls for several interventions for the efficient and environmentally sustainable management of various urban processes, including the road traffic management. Indeed, transportation engineers need to be able to understand how drivers decide their route to effectively address the plethora of challenges for alleviating the congestion phenomena in city areas. In this paper, we model drivers' decision-making with respect to the parking space search, which has been regarded as one of the major causes of traffic congestion. We view the parking search as an instance of sequential search problems and present a game-theoretic investigation of the efficiency of heuristic parking search strategies to locate available parking spot at minimum walking and driving overhead. The analytical study concludes by drawing similarities between the parking game and well-known archetypal games that the Game Theory examines.
\end{abstract}

\section{INTRODUCTION}

To effectively alleviate congestion phenomena in city areas caused by the circulation of large numbers of vehicles in search for available parking space, transportation engineers need to be able to understand how drivers make their decisions concerning route planning and parking spot choices. However, modeling the real-world interaction of drivers' decisions in the emerging smart city environments, admittedly, can be proved to be a very complicated task. On one side the inherent challenging city planning, including the route and parking layout and, on the other side, the uncertainty in the human attitude towards different routing/parking options, induce significant complexity in analytically evaluating the performance of different strategies for route or parking spot selection.

In this paper, we focus on the parking search problem. The general problem of parking space search has seen contributions from different scientific disciplines such as economics, transportation, operations research, and computer science. A number of those assume uncorrelated drivers' decisions and hence, ignore the temporal/spatial correlation in the parking availability patterns. Others take explicitly into account the interactions of drivers' parking search strategies and the associated game-theoretic dimension of the parking search problem.

Formulations of the first case appeared in the context of the broader family of stopping problems. In [1] available parking spots are spread randomly with density $\lambda$ over equal-size blocks that are adjacent to the driver's travel destination. The driver circles through them, crossing the destination every time such a circle is over, and upon encountering a vacant spot he has to decide whether to take it or skip it and seek for a better one. Ferguson in [2] considers a simpler variant of the parking problem, whereby the driver's destination lies in the middle of an infinite-length straight line with parking spots that are occupied with probability $p$. In either case, the optimal policy for the drivers is shown to be of the threshold type: they should occupy an available vacant parking spot whenever this lies within some distance $r=f(\lambda)$, resp. $f(p)$, from their destination and continue searching otherwise. Pricing and the more general economic dimensions of the parking allocation problem are analysed from a microeconomical point of view in [3]. Anderson and de Palma work on a model of stochastic memoryless parking search, i.e., drivers check randomly the availability of spots including those they have already checked; they question whether free access or some pricing structure result in more efficient use of the parking capacity. Likewise, in [4], the authors investigate the pricing effects in the parking problem by treating the availability probabilities of adjacent parking places as statistically independent.

Paradigms of the second case that explicitly accommodate drivers' interaction in game-theoretic models can be found in [5], [6], [7] and [8]. In [5], which seeks to provide cues for optimal parking lot size dimensioning, the drivers decide on the arriving time at the lot, accounting for their preferred time as well as their desire to secure a space. Ayala et al. in [6] define a game setting where drivers exploit (or not) information on the location of others to occupy an available parking spot at the minimum possible travelled distance, irrespective of the distance between the spot and driver's actual travel destination. The authors present distributed parking spot assignment algorithms to realize or approximate the Nash equilibrium states. In a similar study in [7], the informationassisted parking search process is formulated as an instance of resource selection games and a game-theoretic analysis is provided to assess the ultimate impact that different types of parking assistance systems, collecting and sharing information of variable accuracy on parking demand, can have on the parking place selection process. In [8], the authors envisage that drivers use fairly simple heuristics (rules of thumb) to opt for parking choices and investigate which heuristic strategy works well for choosing parking space within an environment whereby the parking availability is shaped by the adopted heuristic strategies (i.e., the availability probabilities of adjacent parking places are not statistically independent). Different simulation scenarios are conducted to derive the equilibrium states in which the competing influences are balanced and no driver has the incentive to change his strategy unilaterally.

This paper comes under the second case, that is, presents a game-theoretic formulation. We consider a simplified structure of a city environment and representative behavioral profiles that help overcome computational hindrances and reduce complexity. In particular, we consider the road topology that 


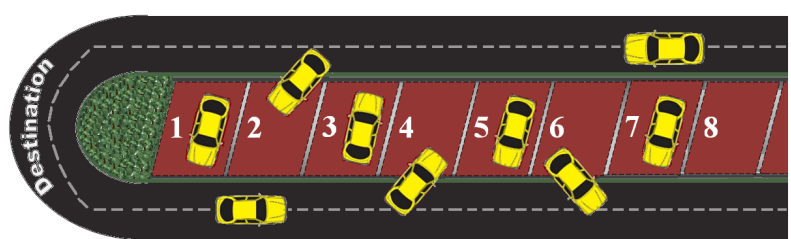

Fig. 1. The structure of the parking environment.

Hutchinson, Fanselow and Todd introduce in [8], that is, a long dead-end street, two one-directional lanes leading to and away from a destination and a parking strip between the two lanes, as shown in Figure 1. As it will become clear in the next section, searching for an empty parking spot in such parking lot arrangement amounts to a type of a sequential search. Typically, empirical evidence shows that decision-makers respond to the complexity of sequential search problems (e.g., mate choice, secretary problem, parking search) by acting heuristically. Interestingly, albeit the human cognitive limitations, time constraints and lack of full information in those reasoning contexts, simple rules of thumb can frequently perform as well as more sophisticated search approaches by exploiting the structure of the information in the environment (ref. ecological rationality in [9]). In this paper, the drivers employ a decision rule based on their distance from the destination, namely the fixed-distance heuristic, which ignores all places until the driver reaches a specific distance from the destination and then takes the first vacant one. Ultimately, we seek to systematically study the efficiency of the fixed-distance heuristic within the sequential parking search context. It is important to notice that this instance of heuristics incorporates two fundamental practices in behavioral decision theory, oneat-a-time processing of pieces of information and the use of thresholds [10]. The fixed-distance heuristic decision strategy is considered in this paper by factoring in knowledge that the driver may or may not have about the status (empty or occupied) of the next to the currently inspected parking spot in the direction towards the destination. This knowledge leads to distinct behaviors and realizations of the empty/occupied spots and thus, two distinct case-studies of the fixed-distance heuristic decision-making: with view-ahead (w-VA) and without view ahead (w/o-VA).

We introduce key concepts and present the assumptions for the parking search environment in Section II. The details of the parking search with and without view ahead and the corresponding game-theoretic analyses are presented in Sections III and IV, respectively. In Section V we focus our attention on the interaction of exactly two drivers and draw similarities between the two-player parking game and wellknown archetypal games that the Game Theory examines. We conclude our study in Section VI.

\section{GAME SETTING}

Implementing the basic assumptions introduced in Section I, we consider a parking lot of $R$ parking spots arranged as shown in Figure 1 and $N$ drivers that seek available parking space within this lot. The drivers employ in their search the fixed-distance heuristic decision rule which ignores all places until the driver reaches as close as $D$ places from the destination and then takes the first vacant one. The collective decision-making on parking place selection can be formulated in the following game setting:
Definition 2.1: A Heuristic-Strategy Parking Game is a tuple $\Gamma(N)=\left(\mathcal{N}, \mathcal{R}, \mathcal{D}, c(k), c^{\prime}(k)\right)$, where:

- $\mathcal{N}=\{1, \ldots, N\}, N>1$ is the set of drivers who seek parking space,

- $\mathcal{R}=\{1, \ldots, R\}, R \geq N$ is the set of parking spots, with set items in increasing order with respect to their distance from the destination (i.e., the closest-to-destination spot is the first set item),

- $\mathcal{D}=\{1, \ldots, N\}$, (recall $R \geq N$ ), is the set of the fixeddistance heuristic strategies with set items that denote at which distance from the destination the drivers initiate their search, - $c(k)$ and $c^{\prime}(k)$ are the cost functions for occupying the $k t h$ parking spot after travelling across the approach lane only, or both lanes, respectively.

In particular, let $a, b, d, e \geq 0$ be the cost weights (more precisely, cost per distance unit, where a distance unit is defined to be the - assumed constant - distance between two consecutive parking spots) for walking $(a)$, driving through the approach lane $(b)$, driving through the return lane $(e)$ and driving away from a particular parking spot (d) (see Fig. 2). For example, $a>b, d$ would mean "prefer driving a bit more rather than walk for long" and $e>b$ would imply "it hurts more if we reach the end of the street and still have not found a spot, hence, have to take a turn and start". Thus, the cost incurred by a driver that parks at the $k t h$ parking spot while travelling in the approach lane is

$$
c(k)=b(R-k)+d(R-k)+2 a k, \quad 1 \leq k \leq R
$$

whereas ending in the same parking spot while travelling in the return lane entails a higher cost, that is

$$
c^{\prime}(k)=b R+e(k-1)+d(R-k)+2 a k, \quad 1 \leq k \leq R
$$

Indeed, the order of a parking event together with the adopted heuristic strategies determine the specific spot at which a driver parks (i.e., the ith parking event, with $1 \leq$ $i \leq N$, occurs at the $k t h$ parking spot) and whether this spot is reached through the approach or the return lane.

In the following two sections, we derive the equilibrium states of the game and assess their (in)efficiency under two distinct case-studies: parking search with and without view of the availability status of the parking spots ahead. As in the original treatment of the problem, we distinguish between the aspiration level (i.e., adopted distance threshold) of a single "mutant" driver $\left(D_{m}, D_{m} \in \mathcal{D}\right)$ and the - assumed to be common - aspiration level of the rest of the population $\left(D_{p}\right.$, $\left.D_{p} \in \mathcal{D}\right)$. Indeed, we seek symmetric equilibria ${ }^{1}$ whereby the expected cost for the mutant driver is minimized at $D_{m}=D_{p}$. The efficiency of the satisficing fixed-distance heuristic strategy is assessed by comparing the cost induced by the equilibrium strategy profiles to that under the optimal parking spot allocation, whereby no driver continues his search in the return lane and hence, the overall cost paid by drivers is minimized. Typically, this is the case when the full information processing and decision-making tasks lie with a centralized parking assistance service.

As last notes, it should be pointed out that the drivers are viewed as decision-makers that, by repeated and varied

\footnotetext{
${ }^{1}$ The derivation of asymmetric equilibria is much harder and their realization in practical situations is much more difficult than that of their symmetric counterparts.
} 


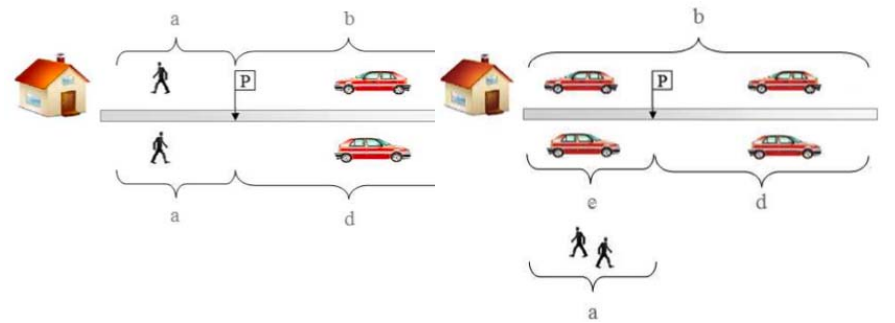

Fig. 2. Cost weights for walking and driving in the approach and return lane, when the driver travels across only the approach lane (left) or both lanes (right) to reach his parking spot.

attempts, adjust their strategy to minimize the incurred cost and hence, they reach those equilibria. In addition, this study implies that all drivers share the same chance of parking at a specific order and none leaves his parking place before the last arrival. This assumption could correspond, for instance, to a scenario where drivers arrive at the business district area in the morning within a given time window, e.g., 8.30-9.00, park for the duration of the working day, and leave the spots in the afternoon to go back home.

\section{FIXED-DISTANCE HEURISTIC PARKING STRATEGY WITH VIEW AHEAD}

In this section we study the fixed-distance heuristic parking strategy in an environment whereby the drivers never occupy a place if the place right next to it is also vacant, on their way to the destination. This is in accordance with the initial formulation in [8] and would correspond to a side-by-side arrangement of parking spots across some street. In such environments the parking spot area fills sequentially, starting from the destination dead-end. Hence, the ith parking event, with $1 \leq i \leq N$, occurs at the $i t h$ parking spot, irrespective of the employed $D_{m}$ and $D_{p}$. However, the corresponding cost does depend on the aspiration level of the driver associated with the ith parking event; namely, the mutant driver pays $c(i)$, if $i \leq D_{m}$ and $c^{\prime}(i)$ if $i>D_{m}$. Figure 3 a plots the cost incurred by the driver associated with the $i t h$ parking event as a function of $i$. By invoking equations (1) and (2), the expected cost of playing $D_{m}$ is given by

$$
\begin{aligned}
E[C]= & \frac{1}{N}\left[\sum_{i=1}^{D_{m}} c(i)+\sum_{i=D_{m}+1}^{N} c^{\prime}(i)\right] \\
= & \frac{1}{2 N} D_{m}\left(1+D_{m}\right)(2 a-b-d)+R(b+d) \\
& +\frac{N-D_{m}}{2 N}\left[\left(1+D_{m}+N\right)(2 a+e-d)-2 e\right]
\end{aligned}
$$

Note that this cost is independent of $D_{p}$. Therefore, in symmetric equilibrium (where all drivers adopt the same strategy) all drivers start their search for parking place once they have reached a distance $D_{m}$ that minimizes the expected cost function in equation (3). Since $\frac{d E[C]}{d D_{m}}<0$, the expected cost is minimized for the maximum value that $D_{m}$ may assume, which is $N$. Thus, starting the search for available parking space from the very beginning, seems the most rational strategy to minimize the expected cost, irrespective of others' preferences (e.g., see Fig. 3b). In terms of Game Theory, this strategy is the best response for all players; namely the game has exactly one (symmetric) equilibrium strategy $D^{e q}=N$. The intuition is that having a view of the next parking place's status brings benefits to the drivers when this is possible (there are empty spots ahead). Hence, by exercising this very a.

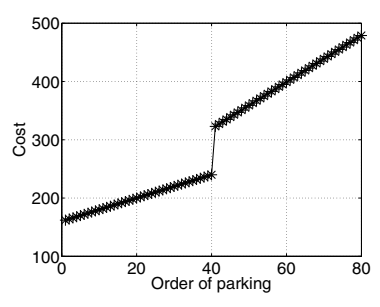

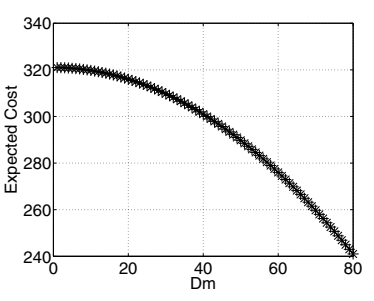

b.
Fig. 3. Parking w-VA: Cost of playing $D_{m}=R / 2$ as a function of the order of parking (left) and expected cost of the fixed-distance heuristic parking strategy (right), $N=R=80, a=2, b=d=e=1$.

simple heuristic strategy at the equilibrium aspiration level $D^{e q}$, drivers will never end up paying the extra penalty of $e$ cost units of driving in the return lane.

\section{FIXED-DISTANCE HEURISTIC PARKING STRATEGY WITHOUT VIEW AHEAD}

In this section, the drivers take the first empty place they encounter within a distance of at most $D$ places from the destination, on their way to the destination. This would correspond to a dispersed yet uniform arrangement of parking spots across a street, so that the status of the next spot is not visible when the drivers reach the immediately previous one. Unlike the case in Section III, the expected cost for using a parking spot depends on both realized strategies $D_{m}$ and $D_{p}$. In order to systematically analyse the consequences of a strategy profile $\left(D_{m}, D_{p}\right)$, we explicitly discriminate between two cases, featuring $D_{m}>D_{p}$ and $D_{m} \leq D_{p}$, respectively.

\section{A. Strategy profiles with $D_{m}>D_{p}$}

In this case, the following observations may be made for the parking patterns (see Fig. 4a):

- drivers (other than the mutant driver) will first fill in all the spots in segment $<$ destination, $D_{p}>$ while approaching the destination, and then start filling up the spots in segment $\left.<D_{p}, D_{m}\right\rangle$ while moving away from the destination. Hence, there is no possibility that the mutant driver encounters an occupied spot in segment $\left\langle D_{p}, D_{m}\right\rangle$ as long as there exists at least one empty spot in segment $<$ destination, $D_{p}>$,

- the mutant driver parks at least $D_{m}$ places away from the destination.

If the mutant driver is associated with the ith parking event, he will park at a distance of $\max \left(D_{m}, i\right)$ spots away from the destination. The corresponding cost is $c\left(D_{m}\right)$, if $i \leq D_{m}$ and $c^{\prime}(i)$, if $i>D_{m}$. Thus, if $D_{m}>D_{p}$ the expected cost becomes

$$
\begin{aligned}
E[C]= & \frac{D_{m}}{N} c\left(D_{m}\right)+\frac{1}{N} \sum_{i=D_{m}+1}^{N} c^{\prime}(i) \\
= & \frac{N-D_{m}}{2 N}\left(1+D_{m}+N\right)(2 a+e-d)+ \\
& \frac{D_{m}}{N}\left[D_{m}(2 a-d-b)+e\right]+R(b+d)-e
\end{aligned}
$$

The analysis of this function gives that $\frac{d E[C]}{d D_{m}}=\frac{1}{N}\left[-D_{m}[2(b-a)+d+e]-a+\frac{d+e}{2}\right]$ and $\frac{d^{2} E[C]}{d D^{2}}=-\frac{1}{N}[2(b-a)+d+e]$. Hence, if $2(b-a)+d+e \geq 0$, then $E[C]$ is concave and monotonically decreasing with $D_{m}$. Otherwise, $E[C]$ is convex. Therefore, the expected cost function assumes its minimum value at

$$
D_{\text {mmin }}=\left\{\begin{array}{l}
N, \text { if } 2(b-a)+d+e \geq 0 \\
\frac{d+e-2 a}{2[2(b-a)+d+e]}, \text { if } 2(b-a)+d+e<0
\end{array}\right.
$$



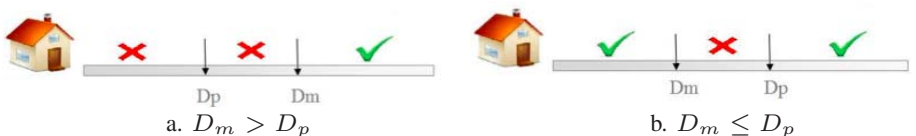

Fig. 4. Parking w/o-VA: Available parking options for a driver with strategy $D_{m}$, given the strategy $D_{p}$ of the rest of the population.

\section{B. Strategy profiles with $D_{m} \leq D_{p}$}

When the mutant driver exposes a more risky behavior comparing to others, the following observations may be made for the parking patterns (see Fig. 4b):

- the drivers fill in sequentially all spots from the parking place $D_{p}$ towards the destination. Hence, there is no possibility that the mutant driver encounters an occupied spot in segment $<$ destination, $D_{m}>$ as long as there exists at least one empty spot in segment $<D_{m}, D_{p}>$,

- the mutant driver parks either at segment $<$ destination, $D_{m}>$ or at least $D_{p}$ places away from the destination.

If the mutant driver is associated with the $i t h$ parking event, it holds that: if $i>D_{p}$, the mutant driver parks at the $i$ th spot at a cost of $c^{\prime}(i)$ units; if $D_{p}-D_{m}<i \leq D_{p}$, he parks at the $\left(D_{p}-i+1\right) t h$ spot at a cost of $c\left(D_{p}-i+1\right)$ units; and if $i \leq D_{p}-D_{m}$, he parks at the $D_{m} t h$ spot at a cost of $c\left(D_{m}\right)$ units. Figure 5 plots the resulting cost of parking events of particular order. Thus, if $D_{m} \leq D_{p}$, the expected cost equals

$$
\begin{aligned}
E[C]= & \frac{1}{N} \sum_{i=D_{p}+1}^{N} c^{\prime}(i)+\frac{1}{N} \sum_{i=D_{p}-D_{m}+1}^{D_{p}} c\left(D_{p}-i+1\right)+ \\
& \frac{D_{p}-D_{m}}{N} c\left(D_{m}\right) \\
= & \frac{N-D_{p}}{2 N}\left[\left(1+D_{p}+N\right)(2 a+e-d)-2 e\right]+R(b+d) \\
& +\frac{D_{m}}{2 N}\left(D_{m}-2 D_{p}-1\right)(b+d-2 a)
\end{aligned}
$$

The analysis of the expected cost function for the monotonicity and concavity trends gives that $\frac{d E[C]}{d D_{m}}=\frac{1}{N}(b+$ $d-2 a)\left(D_{m}-D_{p}-\frac{1}{2}\right)$ and $\frac{d^{2} E[C]}{d D_{m}^{2}}=\frac{1}{N}(b+d-2 a)$. If $b+d-2 a>0$, then $E[C]$ is convex and monotonically decreasing with $D_{m}$, whereas if $b+d-2 a<0$, then $E[C]$ is concave and monotonically increasing with $D_{m}$. (Otherwise, $E[C]$ is a constant function and the driver experiences the same expected cost irrespective of his strategy of preference.) Ultimately, if $D_{m} \leq D_{p}$, the mutant driver minimizes his expected cost if he starts his search for parking space from

$$
D_{\text {min }}=\left\{\begin{array}{l}
D_{p}, \text { if } b+d-2 a>0 \\
1, \text { if } b+d-2 a<0
\end{array}\right.
$$

Hence, the expected cost exhibits one of three possible minimum values ${ }^{2}$, depending on specific conditions on the cost weights. More precisely, by (5) and (7), the expected cost function for the parking search without view ahead assumes its minimum value at

$$
D_{\text {mmin }}\left\{\begin{array}{l}
=N, \text { if } b+d-2 a \geq 0 \\
\in \arg \min _{D_{m^{\prime}} \in\left\{1, \frac{d+e-2 a}{2[2(b-a)+d+e]}\right\}} E\left[C / D_{m}=D_{m^{\prime}}\right], \\
\quad \text { if } 2(b-a)+d+e<0 \\
\left.\in \arg \min _{D_{m^{\prime}} \in\{1, N\} E\left[C / D_{m}=D_{m}\right]}{ }^{\prime}\right] \\
\quad \text { if } 2(b-a)+d+e \geq 0 \text { and } b+d-2 a<0
\end{array}\right.
$$

\footnotetext{
${ }^{2}$ By comparing the left end point $E\left[C / D_{m}=D_{p}+1\right]$ of the branch $D_{m}>D_{p}$, with the right end point $E\left[C / D_{m}=D_{p}\right]$ of the branch $D_{m} \leq$ $D_{p}$, we have that $E\left[C / D_{m}=D_{p}\right]>E\left[C / D_{m}=D_{p}+1\right]$ if $b+d-2 a \geq 0$ and $E\left[C / D_{m}=D_{p}\right]<E\left[C / D_{m}=D_{p}+1\right]$ if $2(b-a)+d+e<0$ and $D_{p}>1$, or if $2(b-a)+d+e<-b$ and $D_{p}=1$.
}
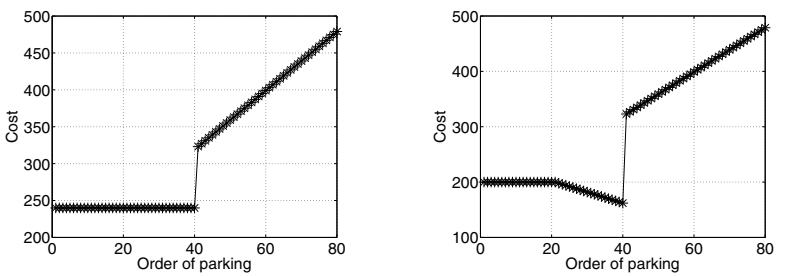

Fig. 5. Parking w/o-VA: Cost of playing $D_{m}=R / 2$ (left) and $D_{m}=R / 4$ (right) against a population who plays $D_{p}=R / 4$ (left) and $D_{p}=R / 2$ (right) as a function of the order of parking, $N=R=80, a=2, b=d=$ $e=1$.

Figure 6 depicts the expected cost for the mutant driver, when he exposes a risk-seeking $\left(D_{m} \leq D_{p}\right)$ or risk-averse $\left(D_{m}>D_{p}\right)$ attitude, given three different values of $D_{p}$. As Figure 6 and equation (4) suggest, all three cases of $D_{p}$ share the same cost results when $D_{m}>D_{p}$. On the contrary, the curves differ on their left part, since the expected cost for $D_{m} \leq D_{p}$ is a function of $D_{p}$ (ref. equation (6)). In all plots the minimum expected costs satisfy the results in (8).

Overall, if we restrict our interest to only symmetric equilibria, the analysis of this section concludes that there is always at most one symmetric equilibrium whereby the drivers demonstrate either a fully conservative attitude, $D^{e q}=N$, starting their search from the beginning (when $b+d-2 a \geq 0$, e.g., see Fig. 6a) or a fully aggressive one, $D^{e q}=1$, anticipating a vacancy adjacent to the destination (when $2(b-a)+b+d+e<0$, e.g., see Fig. 6b). The intuition in the first equilibrium case is that drivers prefer walking than driving and hence, by choosing $D^{e q}=N$ drivers park at the first available parking spot on their way towards the destination, avoiding excess driving in the approach or the return lane. In the second equilibrium case, drivers prefer driving than walking and hence, they are drawn towards spots close to the destination, seeking to minimize the walking overhead. Contrary to the first equilibrium case whereby the simple fixed-distance heuristic rule minimizes the overall social cost, in the second case all but one drivers end up paying the extra penalty of $e$ cost units. In between the two extreme values 1 and $N$, there is no other symmetric equilibrium since there are no cost weights that - satisfy both the conditions $b+d-2 a \geq 0$ (constant or decreasing $E[C]$, for $D_{m} \leq D_{p}$ ) and $2(b-a)+d+e<0$ (convex $E[C]$, for $D_{m}>D_{p}$ ) and - allow the expected cost to exhibit its minimum value at $D_{m}=D_{p}$, with $1<D_{p}<N$.

Finally, when $2(b-a)+d+e \geq 0$ and $b+d-2 a<0$, there is no symmetric equilibrium since the value $D_{m \text { min }}$ decreases with $D_{p}$. In particular, the two possible minimum expected costs that may appear are $E\left[C / D_{m}=1, D_{m} \leq D_{p}\right]$ and $E\left[C / D_{m}=N, D_{m}>D_{p}\right]$. However, the strategy selected by the population, $D_{p}$, affects the minimum expected cost of the mutant driver, only if he demonstrates a risk-seeking behavior, expressed in the strategy $D_{m}=1$. Furthermore, if $D_{m}=1$, the expected cost is a decreasing function of $D_{p}$, since $\frac{d E\left[C / D_{m}=1, D_{m} \leq D_{p}\right]}{d D_{p}}<0$, while it holds that $E\left[C / D_{m}=\right.$ $\left.1, D_{p}=1\right]>E\left[C / D_{m}=N, D_{p}=1\right]$ and $E\left[C / D_{m}=\right.$ $\left.1, D_{p}=N\right]<E\left[C / D_{m}=N, D_{p}=N\right]$. The intuition behind this result is that when the drivers do not have a clear preference over walking or driving (i.e., $2(b-a)+d+e \geq 0$ and $b+d-2 a<0)$ the mutant driver profits from exhibiting the opposite attitude with respect to what all the rest of the population do (see Fig. 6c). Namely, by choosing high (low) $D_{m}$ values under low (high) $D_{p}$ values, the driving (walking) savings counterbalance the walking (driving) overhead. 

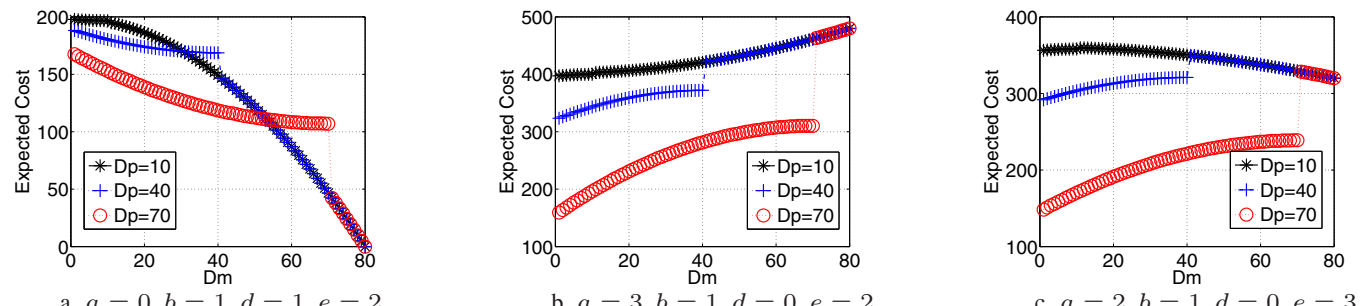

Fig. 6. Expected cost for the fixed-distance heuristic parking strategy w/o-VA, $N=R=80$.

\section{THE TWO-PLAYER GAME $\Gamma(2)$}

In this section we focus our attention on the interaction of exactly two drivers within the reference parking area. Table I shows the resulting game matrix for the strategy profiles.

TABLE I. GAME MATRIX For THE PARKING GAME $\Gamma(2)$.

\begin{tabular}{|c|c|c|}
\cline { 2 - 3 } \multicolumn{1}{c|}{} & $D_{p}=1$ & $D_{p}=2$ \\
\hline$D_{m}=1$ & $A, A$ & $B, C$ \\
\hline$D_{m}=2$ & $C, B$ & $D, D$ \\
\hline
\end{tabular}

We arbitrarily consider the row player as the mutant driver and the column player as the driver that constitutes the rest of the population. With $A, B, C, D$ we denote the expected costs for the row player (or the column player, as they are symmetric) when he responds with $D_{m}=1$ to the population strategy $D_{p}=1$, or with $D_{m}=1$ to $D_{p}=2$, or with $D_{m}=2$ to $D_{p}=1$, or finally, with $D_{m}=2$ to $D_{p}=2$, respectively. By (4) and (6) the expressions for $A, B, C$ and $D$ are

$\begin{array}{ll}A=\left(R-\frac{1}{2}\right)(b+d)+3 a-d+\frac{1}{2} e & B=(R-1)(b+d)+2 a \\ C=(R-2)(b+d)+4 a & D=\left(R-\frac{3}{2}\right)(b+d)+3 a\end{array}$

In order to determine the equilibrium states in the parking game, we compare the expected costs that are induced by either strategy and, hence, define the best responses for every player. Then, we draw similarities between the two-player parking game and well-known archetypal games that present the same equilibria, under the same assumptions for the dominance of the strategies. Thus, it is possible to expand our understanding of the interaction of the drivers by exploiting known results from the theoretic investigation of those classical games (e.g., results regarding the iterative versions of the games).

1) $2(b-a)+d+e<-b$ : Given the particular condition for the costs, we derive that $\mathrm{B}<\mathrm{D}<\mathrm{A}<\mathrm{C}$ and hence, the equilibrium state is $D_{m}=D_{p}=1$. This is an instance of the Prisoner's Dilemma. Therefore, the parking game converges to the "bad" (most expensive) symmetric strategy profile.

2) $b+d-2 a>0$ : This condition results in the ordering $C<D<B<A$, if $2 a+b+e-d>0$ and $C<D<A<B$, if $2 a+b+e-d<0$. This is an instance of the Deadlock Game and, unlike the Prisoner's Dilemma, the action $D_{m}=$ $D_{p}=2$ that is mutually most beneficial (less expensive) is also dominant.

3) $2(b-a)+d+e>-b$ and $b+d-2 a<0$ : These conditions result in $B<D<C<A$ and yield the two asymmetric equilibria $D_{m}=1, D_{p}=2$ and $D_{m}=2$, $D_{p}=1$. This is an instance of the Chicken Game (or HawkDove Game), whereby the players are called to choose whether to back off or risk fighting, with one of the two symmetric strategy profiles (fighting) being disastrous for both. The game, also, exhibits a symmetric mixed-strategy equilibrium where both players randomize over their pure-strategy space.
In this paper, we seek to analytically investigate drivers' decision-making concerning parking spot selection in city environments. The parking search problem has been addressed within the framework of sequential search/optimal stopping problems, whereby decision-makers devise simple heuristic strategies (rules of thumb) to overcome the complexity of finding the optimal decision. In particular, we envisage that drivers use the fixed-distance heuristic according to which drivers start searching for available parking space after they reach as close as a specific distance from their travel destination. Through a game-theoretic investigation, we show that when the drivers are risk-averse (namely, they prefer walking than driving), the simple fixed-distance heuristic strategy leads to optimal parking spot allocation (minimum social cost). Motivated by these results, our intention is to explore scenarios with a richer mix of drivers' preferences for walking and driving, factoring in dynamic scenarios as well, where the drivers leave their parking spots while others still enter the parking area. We expect that this can yield symmetric equilibrium strategies that depart from the fully risky or conservative attitude. The ultimate evaluation of these results would come out of field experimentation, or in a more convenient alternative, dynamic driving emulators that allow for generating the appropriate scenarios and experimenting with real subjects.

\section{ACKNOWLEDGMENT}

This work has been supported in part by EU FP7 EINS under grant agreement No 288021 and the University of Athens (ELKE-10812). The authors would also like to thank Dr. Konstantinos Katsikopoulos from MPI-Berlin for insightful conversations and comments in connection with the internal project COHESION.

\section{REFERENCES}

[1] J. MacQueen and J. Miller, "Optimal persistence policies," Operations Research, vol. 8, no. 3, pp. 362-380, May-June 1960.

[2] T. S. Ferguson, "Optimal stopping and applications," online: http: //www.math.ucla.edu/ tom/Stopping/.

[3] S. P. Anderson and A. de Palma, "The economics of pricing parking," Journal of Urban Economics, vol. 55, no. 1, pp. 1 - 20, 2004.

[4] R. Arnott and J. Rowse, "Modeling parking," Journal of Urban Economics, vol. 45, no. 1, pp. 97 - 124, 1999.

[5] M. Arbatskaya, K. Mukhopadhaya, and E. Rasmusen, "The parking lot problem," Dept. of Economics, Emory Univ., Atlanta, Tech. Rep., 2007.

[6] D. Ayala, O. Wolfson, B. Xu, B. Dasgupta, and J. Lin, "Parking slot assignment games," in Proc. 19th ACM SIGSPATIAL GIS, 2011.

[7] E. Kokolaki, M. Karaliopoulos, and I. Stavrakakis, "Leveraging information in parking assistance systems," IEEE Transactions on Vehicular Technology, vol. 62, no. 9, 2013.

[8] J. M. C. Hutchinson, C. Fanselow, and P. M. Todd, Ecological rationality: intelligence in the world. New York: Oxford Univ. Press, 2012.

[9] D. G. Goldstein and G. Gigerenzer, "Models of ecological rationality: The recognition heuristic," Psychological Review, vol. 109, 2002.

[10] K. V. Katsikopoulos, Advanced guide signs and behavioral decision theory. D. L. Fisher, M. Rizzo, J. K. Caird, and J. D. Lee. Boca Raton, FL: CRC Press, 2011. 